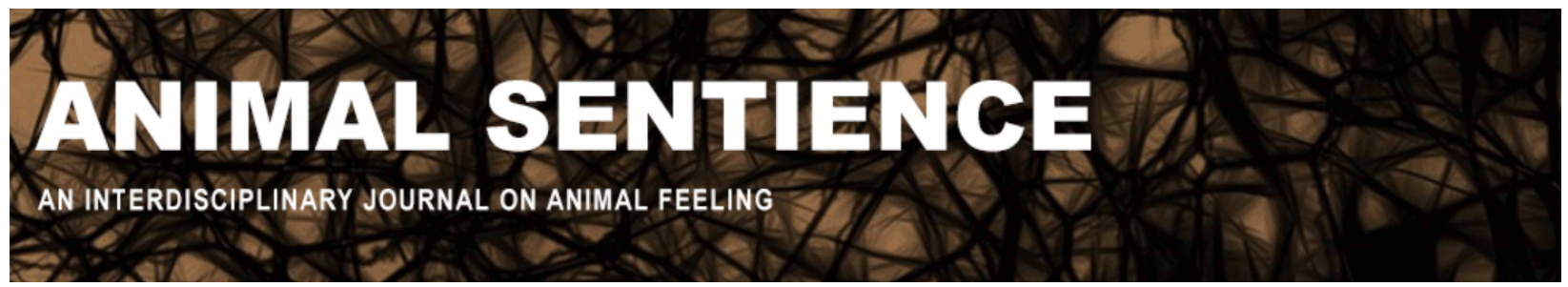

Horowitz, Alexandra; Franks, Becca; and Sebo, Jeff (2018) Fill-in-the-blankemotion in dogs? Evidence from brain imaging. Animal Sentience 22(20) DOI: $10.51291 / 2377-7478.1359$

Date of submission: 2018-07-16 Date of acceptance: $2018-07-20$ (c) 


\title{
Fill-in-the-blank-emotion in dogs? Evidence from brain imaging
}

Commentary on Cook et al. on Dog Jealousy

\author{
Alexandra Horowitz ${ }^{1}$, Becca Franks ${ }^{2}$, and Jeff Sebo ${ }^{2}$ \\ ${ }^{1}$ Department of Psychology, Barnard College \\ ${ }^{2}$ Department of Environmental Studies, New York University
}

\begin{abstract}
What is needed to make meaningful claims about an animal's capacity for subjective experience? Cook et al. (2018) attempt to study jealousy in dogs by placing them in a particular context and then seeing whether they display a particular brain state. We argue that this approach to studying jealousy falls short for two related reasons. First, the relationship between jealousy and the selected context is unclear. Second, the relationship between jealousy and the selected brain state (indeed, any single brain state) is unclear. These and other issues seriously limit what this study can show. It is important not to see this study as showing more than it does.
\end{abstract}

Alexandra Horowitz is adjunct associate professor of Psychology and English and head of the Dog Cognition Lab at Barnard College. She studies the perceptual experience of dogs as well as aspects of the human-dog relationships. Website

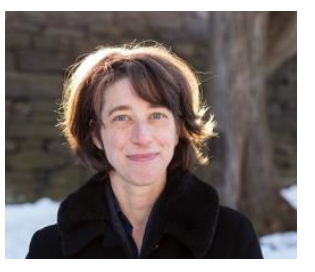

Becca Franks is a visiting assistant professor in the Department of Environmental Studies at New York University. She studies well-being and motivation, with a particular focus on aquatic animal welfare. Website

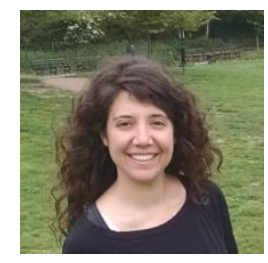

Jeff Sebo is clinical assistant professor of Environmental Studies; affiliated professor of Bioethics, Medical Ethics, and Philosophy; and director of the Animal Studies M.A. Program at New York University. He studies bioethics, animal ethics, and environmental ethics. Website

In order to meaningfully assess whether or not a dog can experience (for example) jealousy, we need at least the following: a clear and distinct definition of jealousy; a context that reliably produces jealousy; and a distinctive outcome (e.g., behavior or brain state) that reliably results from jealousy. With this method, we can then study whether and to what degree the relevant outcome appears in the relevant context.

We should note that this method, although useful, relies on some premises which are unproven: namely, that a context can be reliably jealousy-provoking; that a certain outcome (a 
specific behavior or brain state) will reliably arise from jealousy; and that jealousy is observable in this outcome. This method also risks producing false positives (e.g., the context may produce the outcome for other reasons) and false negatives (e.g., the context may not reliably produce jealousy or jealousy-related outcomes). As a result of these uncertainties, it would be premature to conclude on the basis of any single study that dogs either can or cannot experience jealousy.

With that in mind, we welcome research on this question. Unfortunately, Cook et al.'s (2018) study fails to elucidate the issue at hand. Our particular concerns are as follows: (1) The relationship between jealousy and the selected context is unclear, and (2) the relationship between jealousy and the selected outcome (i.e., blood flow to the amygdala) is also unclear. This of course does not imply that the study is useless; but it does mean that it deviates substantially from the (already limited) ideal. As a result, we can conclude virtually nothing from this study alone. We should accordingly take care not to see it as showing more than it does.

We can trace both the above issues to a previous study (Harris \& Provoust, 2014) whose methods Cook et al. replicate and whose conclusions they treat uncritically. That study was problematic in each of the ways mentioned above. First, a context is created in which a human companion pays attention to a fake dog within view of their companion dog. Yet no evidence is provided that this context reliably produces jealousy - or that it is unlikely to produce other emotional states instead or in addition. Second, an attempt is made to detect jealousy in this context by coding for "aggressive" and attention-seeking behaviors. Yet no evidence is provided that the coded behaviors are likely to result from jealousy or unlikely to result from other emotional states. (Moreover, and notably, attempts to replicate even the weak results from Harris \& Provoust's study have not been successful. Prato-Previde et al., 2018, for example, did not find more attention-getting behavior in this context.) (See also Prato-Previde \& Valsecchi, 2018.)

Consider now how each of these issues arises in the present study. First, regarding the relationship between jealousy and the selected context: the authors create a context in which a human offers food to a fake dog in view of their companion dog. Yet, as other commentaries have also noted (e.g., Serpell, 2018; Vonk, 2018), a dog might reasonably have many non-jealousy reactions in such a situation:

What is that strange thing? (perception of a new object in the environment leads to an interest in investigating the matter further)

Imposter dog! (perception of a new dog-shaped object in the environment leads to an interest in resolving this experience)

There is food going toward a mouth! (perception of food going toward a mouth leads to a desire for food, along with a scarcity response)

There is food coming from a human going toward a mouth! (perception of foodgiving behavior leads to a desire for food along with a scarcity response and foodseeking behavior)

What is even happening right now??? (inability to comprehend what is even happening)

Of course, depending on how the dog perceives the situation, they might also experience jealousy, or a combination of reactions. But given the nature of the situation, it is not clear how dominant 
or immediate we should expect a jealous reaction to be, compared to other reactions. Indeed, a better articulation of the very parameters of jealousy is needed in this and other experimental attempts to elucidate private, phenomenological experiences.

Second, the relationship between jealousy and the selected outcome is also unclear at best. The authors attempt to detect jealousy by observing activation of the amygdala. However, it is far from clear that amygdala activation is necessary for jealousy across species. Even if we all agree, as current scientific practice does, that brain activation - meaning, roughly, more neural activity, as indexed by higher rates of blood flow - is involved in subjective experience, we still have yet to link subjective experience to activation with any level of precision. The closest that researchers have come is the observation that in humans a traumatic loss of certain brain areas can result in a loss of certain abilities. The brain can sometimes compensate for the loss of one area, however, by developing new abilities in other areas. Moreover, when we consider abilities across species, we find that different species can have similar abilities whether or not they have similar brain areas. For example, cephalopods seem able to see without the benefit of a visual cortex (which in humans is necessary for vision; Godfrey-Smith, 2016a,b).

Relatedly, it is far from clear that amygdala activation is sufficient for jealousy across species. In this case, Cook et al. choose to focus on the amygdala because, they say, amygdala activation is involved in "aggression." However, amygdala activation is also said to be involved in sexual attraction, fear conditioning (LeDoux, 1996), assessment of fearful expressions, consolidation of emotional memories (Phelps, 2006), and, according to one of the target article's co-authors, in "arousal ... in response to both positively and negatively valenced stimuli (i.e., either excitement or anxiety)" (Berns et al., 2017). Of course, some of these experiences can occur independently of the others. Thus, either we are wrong to associate amygdala activation with some experiences (which might hence be the case here) or amygdala activation is associated with many kinds of experience (in which case it might or might not be associated with jealousy here).

Of course, Cook et al. are aware of these complications. For example, they explicitly claim that "amygdala activation should not be equated with specific emotions." Yet the validity of any inference would still depend on the existence a sufficiently reliable relationship between jealousy and the amygdala. Here one wonders: If the amygdala had received less blood flow, would that have been evidence that dogs cannot experience jealousy? If a different region had received more blood flow, would that have been evidence that dogs can experience jealousy in a different region of the brain (or merely a lack of evidence that they cannot)? Without a clear definition at the appropriate level of analysis (in this case, experiential/psychological), changes at other levels of analysis (i.e., anatomical/neurobiological) risk becoming irrelevant until proven otherwise.

Here the authors might object that amygdala activation can be correlated with jealousy whether or not it is necessary or sufficient for jealousy-and that if it is, then it can still be evidence of jealousy. However, our point is not that amygdala activation would have to be necessary or sufficient for jealousy in order to count as evidence of jealousy. Our point is that the correlation between amygdala activation and jealousy is currently so unclear that even calling the former evidence of the latter (especially in such a contrived context) risks overstating its importance.

An overstated positive result could do clear harm, for example, by leading people to think that dogs are having more human-like experiences than they are and leading people to treat dogs in more human-like ways than they should. An overstated negative claim could also do harm, for example, by allowing people to treat dogs callously, assuming that they are not experiencing 
certain kinds of emotion at all. This is part of why it matters so much that we get the methodology right - not only because of its implications for this study, but also because of the precedent that it sets for future studies, a sentiment echoed in other commentaries on this target article (e.g., Adolphs, 2018; Silver \& Santos, 2018).

One separate methodological point: Cook et al.'s analysis is based on the activation patterns in only one group of dogs, an "aggressive" group, based on a behavior inventory, the CBARQ, which asks humans to rate dogs' responses in various contexts. Supplemental information indicates that six dogs did indeed score slightly higher than the other seven; whether this difference is significant (statistically or otherwise) is not discussed. These six dogs also showed higher rates of excitability, food-stealing, and attention-getting than the other dogs; the implications of this for the interpretation of the amygdala activation data are not discussed.

Cook et al.'s work adds to a long and complicated history of scientific research hoping to elucidate the subjective experience of nonhumans: animal cognition. The scientific study of animal minds has come a long way over the last half-century. Results in this field can have important ramifications for an improved relationship between humans and other animals. We are optimistic that with further careful work in this field, progress will continue to be made.

\section{References}

Adolphs, R. (2018). What would we like to know by imaging the brains of dogs?. Animal Sentience 22(14).

Berns, G. S., Brooks, A. M., Spivak, M., \& Levy, K. (2017). Functional MRI in awake dogs predicts suitability for assistance work. Scientific Reports 7, 43704.

Cook, P., Prichard, A., Spivak, M., \& Berns, G. S. (2018). Jealousy in dogs? Evidence from brain imaging. Animal Sentience 22(1).

Godfrey-Smith, P. (2016a). Other Minds: The Octopus, the Sea, and the Deep Origins of Consciousness. Farrar, Straus and Giroux.

Godfrey-Smith, P. (2016b). Pain in parallel. Animal Sentience 3(21).

Harris, C. R., \& Prouvost, C. (2014). Jealousy in dogs. PLoS ONE 9(7), e94597.

LeDoux, J. E. (1996). The Emotional Brain. New York: Simon \& Schuster.

Phelps, E. A. (2006). Emotion and cognition: Insights from studies of the human amygdala. Annual Review of Psychology 57, 27-53.

Prato-Previde, E., \& Valsecchi, P. (2018). What is it like to be a jealous dog? Animal Sentience 22(16).

Prato-Previde, E., Nicotra, V., Pelosi, A., \& Valsecchi, P. (2018). Pet dogs' behavior when the owner and an unfamiliar person attend to a faux rival. PLOS ONE, 13(4), e0194577.

Serpell, J. A. (2018). Jealousy? or just hostility toward other dogs? The risks of jumping to conclusions. Animal Sentience 22(13).

Silver, Z. A., \& Santos, L. R. (2018). Understanding dogs' neural responses in a food-giving paradigm. Animal Sentience 22(15).

Vonk, J. (2018). Researchers, not dogs, lack control in an experiment on jealousy. Animal Sentience 22(2). 